\title{
The Multi-Stimulus Box: An innovative learning device for the comparative study of object perception and recognition with various types of stimuli
}

\author{
Michael Morten Steurer • Claudia Stephan • \\ Josef Aringer • Wolfgang Berger • Ulrike Aust
}

Published online: 5 January 2012

(C) Psychonomic Society, Inc. 2011

\begin{abstract}
In this article, we report the construction of a novel type of automated learning device for exploring a broad range of issues in animal visual cognition. The testing box (MultiStimulus Box, or MSB) we describe is an experimental chamber that enables the flexible presentation of various stimulus types while providing control over incidental cues to the greatest possible extent. Among the stimuli that can be presented are photographs, real objects, and even holograms. The MSB allows for comparative research across different stimulus qualities and species, and is thus a promising tool for advancing our understanding of the role of stimulus qualities for animals' perception, discrimination, and categorization of objects.
\end{abstract}

Electronic supplementary material The online version of this article (doi:10.3758/s13428-011-0179-4) contains supplementary material, which is available to authorized users.

M. M. Steurer $(\square) \cdot$ C. Stephan $\cdot$ U. Aust

Department of Cognitive Biology, University of Vienna,

Althanstraße 14,

1090 Vienna (Wien), Austria

e-mail: michael.steurer@univie.ac.at

M. M. Steurer

Aerosol Physics and Environmental Physics,

University of Vienna,

Strudlhofgasse 4,

1090 Vienna (Wien), Austria

J. Aringer

Physics of Functional Materials, University of Vienna,

Strudlhofgasse 4,

1090 Vienna (Wien), Austria

W. Berger

Messerli Research Institute, University of Veterinary

Medicine Vienna, Medical University Vienna,

University of Vienna,

Veterinärplatz 1,

1210 Vienna (Wien), Austria
Keywords Multi-Stimulus Box · Animal cognition · Holograms · Objects $\cdot$ Photographs

Comparative research requires not only testing different species under comparable experimental conditions, but also testing a particular species (or even the same individual) with different types of stimuli. For example, our knowledge of how animals perceive two-dimensional (2-D) pictures as compared to real, three-dimensional (3-D) objects is still far from complete (see, e.g., Aust \& Huber, 2006; Cook \& Katz, 1999; Loidolt, Aust, Steurer, Troje, \& Huber, 2006). Other stimulus types, like holograms, have not yet been tried in studies on animal cognition, although considerable benefit can be expected from using such types of representations. Like real objects, holograms provide full 3-D shape information, and may thus be a powerful means for investigating the extent to which the third dimension supports recognition and categorization. But, relative to real objects, holograms bear the advantage of offering many possibilities of systematically modifying the appearance of a stimulus (for more details on holography, see, e.g., Ackermann \& Eichler, 2007; Collier, Burckhardt, \& Lin, 1971; Saxby, 2004).

One major reason why research involving different stimulus qualities is still sparse is the fact that such experiments are highly challenging from a technical point of view. Namely, an apparatus is required that can allow for the presentation of different types of stimuli while, at the same time, keeping extrinsic factors equal (or modified in the same way) across stimulus types. Previous attempts to provide such an apparatus had been strongly limited regarding the range of stimulus qualities and the number of stimuli that could be presented (see, e.g., Friedman, Spetch, \& Lank, 2003), and for the most part, such devices were unable to 
eliminate incidental cues to a satisfactory extent. Such cues could, for example, be time patterns that inadvertently emerged in the course of stimulus presentation, changes in stimulus background or in the direction and intensity of incident light, or background noises or magnetic fields induced by movements of (parts of) the apparatus that occur when the stimuli are presented.

The development of the apparatus described here was thus motivated by the need for a method to explore the perceptual and cognitive abilities of animals in general, and pigeons in particular. Namely, we aimed at devising a technically elaborate apparatus that would allow for the flexible presentation of various stimulus types (such as photographs, objects, and holograms) while providing control over extrinsic factors to the greatest possible extent. The latter requirement included, on the one hand, efficient elimination of incidental cues that might lead to artifacts in the animals' responding behavior. On the other hand, we aimed at controlling and systematically varying such factors as illumination (color, direction, and intensity), perspective (viewing angle), movement (static and dynamic presentation), or acoustic signals, in order to investigate their possible roles in animal learning and categorization. Our ultimate goal was thus to devise an apparatus that would allow for comparative research across different stimulus qualities and presentation conditions. Furthermore, the box-although originally devised for pigeons - can be modified for use with other species as well, thereby enabling cross-species comparisons under almost identical experimental conditions. We expect the versatility of this elaborate and sophisticated apparatus to open up a wide field of research possibilities, and thus to contribute to increasing our knowledge on aspects of animal visual cognition that have, so far, remained unresolved. For reasons of conciseness, we will refer to our apparatus as a Multi-Stimulus Box (MSB) in the remainder of this article.

\section{The Multi-Stimulus Box}

Basic design and functional principle

Figure 1 gives a schematic overview of the basic design and functional principle of the MSB (for details of the box as presently in use in our laboratory, see Fig. 3). The innovative idea of the MSB, which distinguishes it from traditional types of experimental chambers, is to present stimuli attached to individual "slots" that are mounted onto a vertically rotating wheel. When the wheel stops, one of the stimuli fixed to the wheel becomes visible through a clear Perspex window (a "pecking key") to the animal, which is placed in a closed Skinner box. The distance between the stimulus and the pecking key is bridged by a viewing tunnel made of dark opaque material, whose length restricts the subject's viewing angle. Thus, its view onto stimuli placed in neighboring slots is obstructed. A black roller blind behind the wheel serves as a (common) background for all presented stimuli.

The "slot machine" principle solves the problem of different backgrounds with different stimuli (which is encountered with apparatuses in which the individual stimuli are placed in different chambers), because the background against which the stimuli are presented always stays the same. To avoid any temporal and acoustic incidental cues possibly emerging through swapping slots (i.e., stimuli), the wheel is always turned in the same direction and, by means of extra turns, for an additional (pseudo)random period of time. This makes the durations of intertrial intervals (ITIs) unpredictable and devalues possible discriminative cues such as noise accompanying the process of exchanging the stimuli to be presented (such as clicking relays, motor noises, etc.), as well as other potential influences such as magnetic fields. Such incidental cues are often a problem in setups in which stimuli (or stimulus chambers) are attached to a slider that is moved left and right in order to swap
Fig. 1 Schematic drawing of the basic design of the MultiStimulus Box (side view)

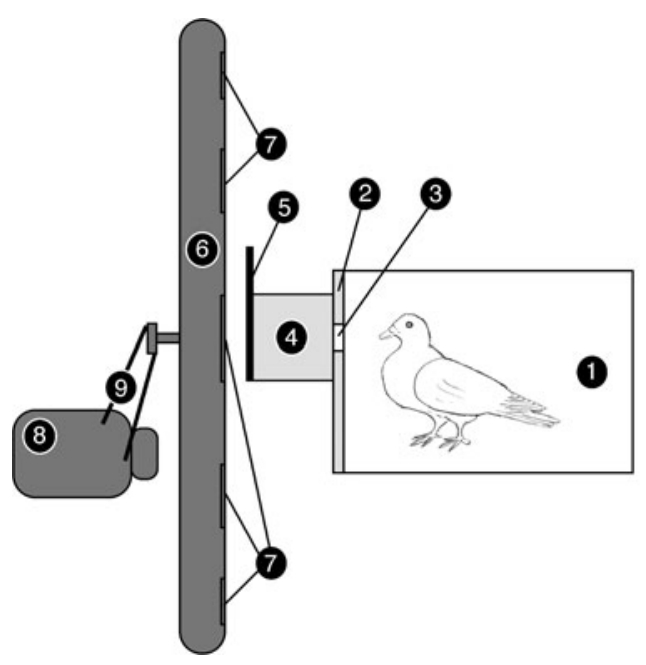

() Skinner-box with pigeon

Intelligence panel

3 Pecking key

(4) Viewing tunnel

(5) Shutter

6 Wheel

Slots for stimuli

8 Stepper motor (for wheel rotation)

(9) Toothed belt (connects 8 with 6) 
stimuli (e.g., Friedman et al., 2003). Namely, such undesired incidental cues may vary for different sliding directions, thereby possibly serving as signals for the forthcoming presentation of particular stimuli. It is noteworthy that the MSB does not eliminate noise and magnetic fields, but rules out the possibility of them being used as discriminative cues, because the wheel always turns in the same direction at the same speed prior to every stimulus presentation. As a consequence, any incidental cues are the same in every trial.

Also, the maximum moving time of a slider is limited by the distance between its two extreme positions, which is not a problem with a wheel. Although sliders may be moved at different speeds in order to alleviate the problem of temporal cues, this is possible only within narrow limits and may also be challenging from a technical point of view. Furthermore, the distances between extreme positions are normally quite short in (spatially constrained) laboratory settings, so that the effects that such variations in moving speed have on the actual duration of ITIs may only be modest. With the MSB, by contrast, ITIs can be prolonged to almost any desired duration simply by adding further wheel rotations, and without any changes in speed being necessary.

The basic concept of the MSB can be extended by adding further wheels that are placed one behind the other to allow for the use of different types of stimuli. For example, one wheel may be equipped with holograms and another one with real objects. Furthermore, the use of two or more wheels allows for providing different distances of the presented stimuli from the observer, or for presenting compound stimuli whose constituent parts are shown on different wheels. For example, the effects of partial stimulus occlusion might be investigated by placing stimuli on one wheel and potential occluders on the wheel in front. Also, the appearance of stimuli placed on the back wheel(s) could be modified by filters (e.g., color/wavelength, polarization) inserted into the slots of the wheel in front.

Furthermore, the appearance of the stimuli can be modified independently from the wheel system described so far. For example, extrinsic factors such as acoustic cues or perspective (i.e., rotation of the stimuli) can be manipulated. Illumination can be changed by using different light sources that vary in intensity, direction, wavelength, and so forth. To distinguish between these two - technically different-possibilities of controlling stimulus appearance, we refer to them as wheelassociated and wheel-independent stimulus modifications.

As soon as all settings have been made (including wheel positions, illumination, rotation angle, etc.), stimulus presentation is started by a shutter being raised. When the (predefined) presentation time is over, the shutter is closed again. Therefore, the experimental subject's view onto the presentation unit is obstructed during the ITI, so that the subject cannot observe the transitions between stimuli and/ or settings.
General operations protocol

The protocol defined for operating the MSB provides both flexibility and easy handling regarding control of the apparatus and the procedure of the experimental sessions. The different levels of technical realizations are separated, with the software and experimenter representing the highest level. Assignment of the individual stimuli to the available slots is accomplished at this highest level, which ensures control over the stimulus positions. Also, the number of wheel rotations between stimulus presentations (determined by the number of "steps" from slot to slot) is defined at this level.

The next level below is that of the communication protocol, which is used by the experimental software to transmit commands to the executing parts of the apparatus. Thereby, the wheel-associated and wheel-independent systems are generally addressed separately. A special command is needed to bring all wheels into a predefined starting position at the beginning of each experimental session. By means of a data transfer command, the wheel-associated system is loaded with information about the number of slot steps each wheel has to perform in order to proceed from the current slot position to the next one that is specified in the program. ${ }^{1}$ A further command is needed to start turning all wheels according to the previously specified number of slot steps, as well as a command for aborting all current movements or any incomplete data transmission. Communication with the wheel-independent system follows the same logic. A special command is used for initialization (i.e., to bring the system to a predefined state), another command loads the apparatus with information about the settings for accessory devices (such as light sources), and a start command instructs the system to perform all coded actions. Again, a special command is needed for aborting all current actions or any incomplete data transmission. Additional commands are available for instructing the system to start or end presentation (i.e., to open or close the shutter), as well as an emergency halt command to abort the entire session (i.e., to stop all procedures). A complete list of commands at the communication protocol level is provided in the supplemental materials.

By means of three binary flags, the system gives feedback to the software (and thus the user) about its current internal state: that is, if it is free or busy processing a command, and whether the initialization of its parts - the wheel-associated and wheel-independent ones-has been successful. When initialization is completed, the experimenter can insert the stimuli into the appropriate slots. The software then commands the system to make the stimulus

\footnotetext{
${ }^{1}$ For example, the command "WHEEL_DATA_CMD 19, 8" would mean 19 slot steps on Wheel 1 and 8 slot steps on Wheel 2. If there were, for instance, nine slots on each wheel, this would effectively code a shift to the subsequent slot on Wheel 1 by means of two extra turns and a shift to the slot preceding the current one on Wheel 2.
} 
settings for the first trial (see last paragraph), and starts the presentation (by means of the start presentation command). After the presentation has been terminated (by means of the end presentation command), a new trial can start. Due to this very general protocol, any apparatus of this type could be controlled in the same way-no matter how many slots or wheels it might have and no matter which extrinsic factors would be manipulated.

At the electronic level, a 1-Wire ${ }^{2}$-based terminal device was used for realizing the communication protocol, in order to make it compatible with all our other laboratory technology, which is based on 1-Wire buses as well. Namely, we utilized a DS2408 chip (see http://www.maxim-ic.com), which can be used for communication with microcontrollers or other electronic devices. The chip provides eight bits, which can simultaneously be read or written onto. Four bits are used for flag signals, and the other four serve either for coding a command or for data transmission (see Fig. 2). More details are provided in the supplemental materials.

The apparatus realized in our laboratory

What follows is a more detailed description of the box as it is presently realized in our pigeon laboratory. Figure 3 gives an outside view of the MSB. The external measures of the apparatus are $90 \times 150 \times 146 \mathrm{~cm}^{3}$ (depth $\times$ width $\times$ height). It rests on four aluminum posts $(50 \mathrm{~cm}$ tall $)$ with wheels for moving the apparatus, if necessary. The casing consists of an aluminum frame and plastic panels. This cladding is opaque and helps to protect the inner parts of the apparatus against dust and dirt. The right and left side panels are doors that help with servicing the box. The front panel contains the cutout for the "viewing tunnel" (at the end of which the stimuli are presented) with a removable intelligence panel in front. The latter includes a translucent pecking key $(\varnothing 5 \mathrm{~cm})$. Furthermore, the front of the box possesses a docking system for a mobile Skinner box (which contains a loudspeaker but no intelligence panel). The outer measures of the Skinner box are $53 \times 42 \times 45 \mathrm{~cm}^{3}$. The feeder for administering a reward is situated below the intelligence panel, and it consists of a motor that lifts a piston up through a food reservoir and a hole in the bottom of the Skinner box in order to make the grain, which accumulates in a depression at the top of the piston during its way through the food container, available to the animal. The animal's behavior during experimental sessions can be monitored by means of a surveillance camera that is mounted inside the Skinner box.

Figure 4 provides an inside view of the MSB. A construction sketch showing the main parts of the stimulus presentation system can be found in the supplemental

\footnotetext{
${ }^{2} 1$-Wire is a registered trademark of Maxim Integrated Products, Inc.
}

materials (Fig. S1). So far, we have only used single stimuli (i.e., no compound ones that would require more than one wheel for all parts being presented), and only two wheels in total - one in front, which currently serves for presenting transmission holograms, and one behind, for showing either real objects or photographs. Thereby, the holograms project onto the same spot at which the real objects and photographs are shown, so that all stimuli are indeed perceived in the same position by the observing animal. A stimulus example is shown in Figure 5.

The wheels are operated by means of two stepper motors, with two toothed belts connecting the wheels to the motor shafts. Each wheel completes a full rotation in $7.5 \mathrm{~s}$ on average (because wheels have to be accelerated at the beginning and slowed down at the end of the movement). The front wheel is $80 \mathrm{~cm}$ in diameter. The back wheel, which is located $15 \mathrm{~cm}$ behind, is $30 \mathrm{~cm}$ in diameter. Each wheel has nine "slots" for mounting the stimuli to be presented. The back wheel has nine 20-cm-long arms that extend from the central disc, with one slot being located at the end of each arm. By contrast, the slots are directly integrated into the front wheel, which allows for inserting, for instance, hologram plates.

The gap between the front wheel and the pecking key (or any other viewing window that may be inserted to adjust the device for use with other species) is bridged by the viewing tunnel, which is $10 \times 10 \times 8 \mathrm{~cm}^{3}$. The shutter is situated between the front wheel and the tunnel. The total distance between the pecking key/viewing window and the front wheel is therefore about $11 \mathrm{~cm}$, and the distance between the pecking key/viewing window and the back wheel is about $26 \mathrm{~cm}$. In our present setup, this equals the viewing distance for all three stimulus types (real objects, photographs, and holographic images). This may be relevant regarding the finding that distances beyond 6-7 $\mathrm{cm}$ may already begin to tax the capacity of pigeons' frontal field (see Cook, 2001), which is probably used for binocular, myopic viewing, and is thus assumed to be specialized for examining fine local details. Instead, pigeons may be more prone to using their lateral fields then, which are probably dedicated to more global, wide-field perception (see, e.g., Bloch \& Martinoya, 1982; Bloch, Rivaud, \& Martinoya, 1984; Cook, 2001; Jäger \& Zeigler 1991). Therefore, our experimental setup may favor global processing and may thus produce different results than have many experiments carried out in traditional Skinner boxes, which have yielded evidence of a local precedence effect in pigeons (e.g., Cavoto \& Cook, 2001; Cerella, 1980).

The maximum size of a real object that can be inserted (and will be fully visible) is about $7.5 \times 10 \times 10 \mathrm{~cm}^{3}$. Accordingly, photographs can be up to $10 \times 10 \mathrm{~cm}^{2}$ (width $\times$ height). The slots in the front wheel are $12.5 \times 24.5 \mathrm{~cm}^{2}$, which equals the size of the hologram plates that are inserted. The slots consist of a frame and a window of $10 \times 8 \mathrm{~cm}^{2}$, through which the 


\section{Control Computer}

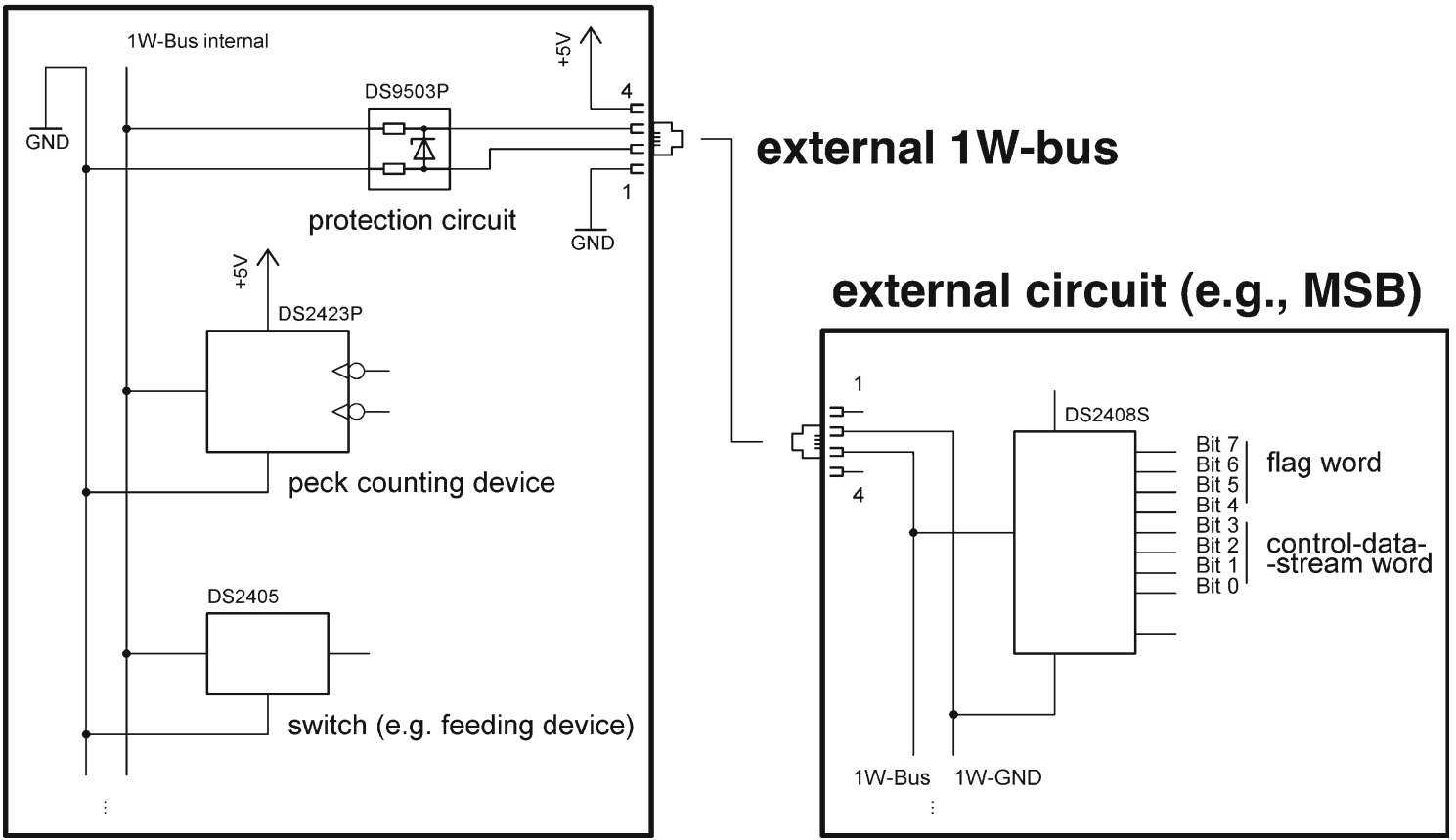

Fig. 2 Schematic drawing of the 1-Wire-driven components of the control computer (left) and the Multi-Stimulus Box (MSB) electronics (right)

stimulus is visible. The maximum size of the holographic images is the same as for real objects, since they project onto the same spot at which objects are presented.

Except when compound stimuli are presented, at least one slot of the front wheel should remain empty during experiments in order to ensure an unblocked view of the stimuli presented on the back wheel. Likewise, one or more slots should remain empty on the back wheel in order to allow for undisturbed presentation of stimuli shown on the front wheel (i.e., without a second stimulus being visible behind, on the back wheel).

The number of stimuli that may be presented within a session without repeating individual stimuli is limited to eight if only one wheel is used. If both wheels are used, 16 different stimuli per session can be presented, and if compound stimuli whose parts are presented on different wheels are shown, a total of 81 different stimuli (i.e., combinations of slots on the front and back wheels) are possible, as no empty slots would
Fig. 3 Outside view of the MSB (back wall of the Skinner box removed)



(1) Control computer

(2) Control monitor

(3) Main switch

(4) Emergency switch

(5) Experimental chamber (Skinner-box)

6) Intelligence panel with pecking key

(7) Surveillance camera

8 Lid of Skinner-box (opened)

9) Feeder

(10) Laser control device

(11) Hologram storage

12 Side doors (for servicing and repairs) 


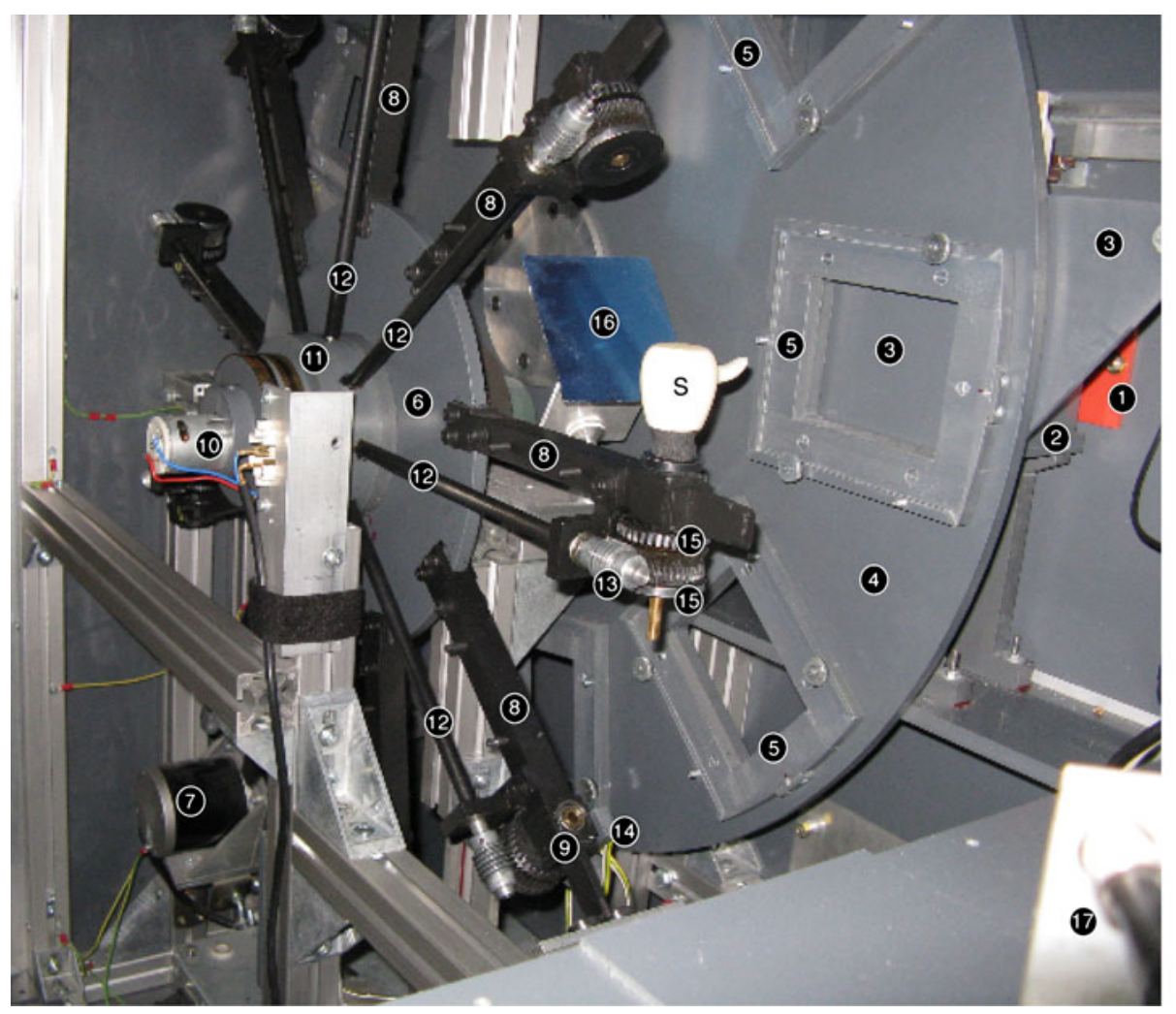

(1) Intelligence panel with pecking key

(2) Viewing tunnel

(3) Shutter

(4) Front wheel (holograms)

(5) Slots (for holograms)

6 Back wheel (objects/photographs)

$(7$ Stepper motor (for back wheel rotation)

8 Arms (for objects/photographs)

(9) Device for mounting stimuli

(10) Engine (for object rotation)

(11) Bevel gear (for object rotation)

(12) Shaft

(13) Snail wheel transmission unit (for object rotation)

(14) IR-sensors (for object rotation system)

(15) Light-reflecting wheels (for orienting the object)

(16) Mirror (for object illumination)

17 Laser beam outlet (hologram illumination)

S Stimulus

Fig. 4 Inside view of the MSB

be required then. Nevertheless, this may still seem too few stimuli for experiments in which open-ended categorization with large numbers of stimuli would be investigated. It should, however, be considered that even such tasks could be conducted by exchanging stimuli between sessions. In short, the number of stimuli that can be presented without repetition in a session is limited, but the number of stimuli that can be presented in a particular task is not.

Regarding the wheel-independent system, we have implemented various modes of illumination. For illumination of real objects and photographs, as well as for replaying the holograms, a green diode-pumped solid-state laser (DPSS) with a wavelength of $532 \mathrm{~nm}$ is used, thus providing light of the same quality (e.g., wavelength) in all presentation modes. The laser intensity can be adjusted with the laser device electronics. The laser light is split into three beams, one for hologram replay and two for object (or photograph) illumination. These beams are then sent through neutral-density filters. Several filters are mounted onto a circular disc, and the MSB control electronics chooses from these the filter prespecified by the executing software. The laser beams are injected into optical fibers whose outlets are mounted in the appropriate

Fig. 5 Stimulus examples: A real object (left) and its presentation as hologram (right). The hologram is seen from the Skinner box through the viewing tunnel. (The intelligence panel with the pecking key has been removed.)

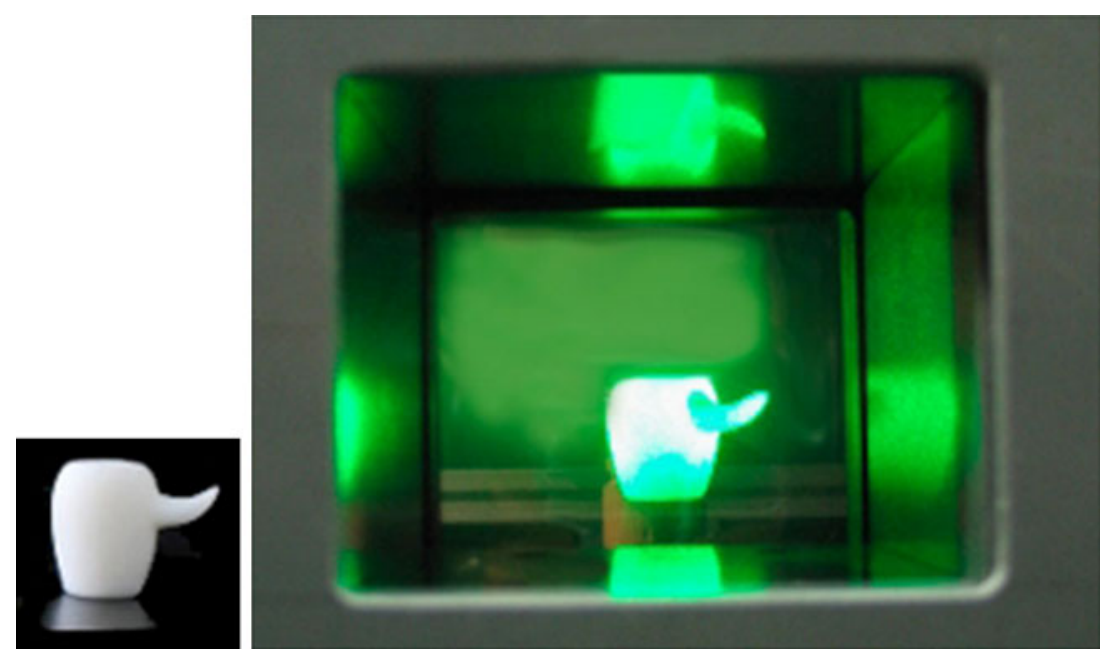


positions for correctly illuminating the stimuli. For safety reasons, the MSB is equipped with door-controlled interlock circuits that switch off the laser source as soon as one of the doors of the MSB is opened.

Also, the wheel-independent system provides the possibility of showing objects in motion. During presentation, they either can remain in the orientation they had been brought into prior to presentation (static stimuli; 36 possible positions) or can be rotated (dynamic stimuli). Regarding dynamic presentation, two rotation modes are possible: Stimuli may turn to either the right or left between two predefined positions, or may continuously rotate in either a clockwise or counterclockwise direction. Of course, the rotation system can also be turned on when static objects-like holograms or photographs - are presented, in order to provide the same background noise as during dynamic presentation.

\section{Software}

All experiments on our apparatus are controlled by CognitionLabLight, a software system programmed by the first author. The software loads experimental settings from a so-called $P O L$ file, in which general information about the parameters of the procedure (go/no-go) and a list with stimulus definitions for each trial are provided. An example of a POL file is provided in the supplemental materials. Stimuli can be given arbitrary names, and the software decides by means of pattern matching to which wheel a particular stimulus belongs. The software reads the whole file and assigns the stimuli to the correct wheels at the onset of the session. Assignment to the individual slots of a wheel is pseudorandom. For each stimulus to be mounted, the program brings the wheel into the appropriate position and asks the experimenter to attach the correct stimulus. The session starts when all of the stimuli are mounted. During the ITI, the software orders the MSB control electronics to conduct a particular (random) number of slot steps and then to stop when the correct combination of wheel positions for the next trial has been reached. The wheel-independent settings for each stimulus are coded in the trial list after the stimulus name as a binary sequence of numbers (0/1). Alternatively, textual abbreviations can be defined and used in order to increase clarity (e.g., green-object-clockwise instead of 00001000-0000011111; see the supplemental materials for more information). The program logs all pecks emitted by the pigeon and controls the entire experimental session, without any interference by the experimenter being required.

\section{Summary and outlook}

The MSB represents an innovative method for exploring the perceptual and cognitive abilities of animals that bears a number of advantages over traditional setups. First, it allows for the flexible presentation of various stimulus types under otherwise identical experimental conditions, and thereby provides maximum comparability of results. Among the stimuli that can be shown are photographs, real objects, and even holograms. The latter have never been used in animal visual experiments before, though they may substantially advance our understanding of how animals process visual information. Second, incidental cues given, for example, by magnetic fields or noises caused by the apparatus can largely be eliminated with the MSB. Finally, the MSB provides the possibility of varying such factors as stimulus illumination, perspective, and movement, and thus allows for systematically investigating their effects on performance.

These features make the MSB a promising means for reinvestigating a number of tasks in which pigeons (and other animals) have failed so far, or that have yielded ambiguous results, with traditional methods. In particular, many methodological problems that may have prevented animals from succeeding in traditional setups can be eliminated in the MSB. A major field of application for the MSB would, for instance, be tasks in which pigeons may have failed in previous experiments due to the two-dimensionality of the employed stimuli. For example, it may be worth reinvestigating the longstanding question of whether pigeons are able to perceive the unity of partly occluded figures by means of real, 3-D stimuli (e.g., Aust \& Huber, 2006; see Ushitani \& Fujita, 2005). In the MSB, the birds could be confronted with compound stimuli, consisting of objects fixed to the back wheel and partial occluders placed on the front wheel. As the perception of two planes (those of the occluder and of the occluded object) is a prerequisite for perceptual completion, such a setup could be more likely to reveal such an ability than are "flat" 2-D stimuli, which might not lead to the perception of depth, and thus to the perception of object unity, in pigeons. Similarly, the role of motion for stimulus recognition and classification could be investigated more efficiently with the MSB than has been the case previously (see, e.g., Cook \& Katz, 1999; Loidolt et al., 2006). For example, it might be promising to assess performance on static and dynamic objects and holograms and to compare the results with those obtained with animated (2-D) images shown on a computer screen.

In summary, the development of the MSB provides a substantial step forward in understanding the role of stimulus quality for pigeons' perception, discrimination, and categorization of objects. We are confident that the versatility of the MSB will open up a wide field of research possibilities and thus will increase our knowledge of aspects of pigeon visual cognition that have, so far, remained controversial. In addition, the fact that the 
MSB may also be used with a variety of different species will further increase the number of its possible fields of application and its value for comparative cognition research.

Author Note Development and implementation of the MSB were supported by a grant from the Austrian Science Foundation (FWFP20240) to U.A. Thanks are due to Peter A. Hoffmann for discussion and technical advice.

\section{References}

Ackermann, G., \& Eichler. (2007). Holography: A practical approach. WILEY-VCH: Weinheim.

Aust, U., \& Huber, L. (2006). Does the use of natural stimuli facilitate amodal completion in pigeons? Perception, 35, 333-349.

Bloch, S., \& Martinoya, C. (1982). Comparing frontal and lateral viewing in the pigeon: I. Tachistoscopic visual acuity as a function of distance. Behavioural Brain Research, 5, 231-244. doi:10.1016/0166-4328(82)90031-6

Bloch, S., Rivaud, S., \& Martinoya, C. (1984). Comparing frontal and lateral viewing in the pigeon: III. Different patterns of eye movements for binocular and monocular fixation. Behavioural Brain Research, 13, 173-182. doi:10.1016/0166-4328(84)90147-5
Cavoto, K. K., \& Cook, R. G. (2001). Cognitive precedence for local information in hierarchical stimulus processing by pigeons. Journal of Experimental Psychology: Animal Behavior Processes, 27, 3-16.

Cerella, J. (1980). The pigeon's analysis of pictures. Pattern Recognition, $12,1-6$.

Collier, R., Burckhardt, C., \& Lin, L. (1971). Optical holography. New York, NY: Academic Press.

Cook, R. G. (Ed.). (2001). Avian visual cognition [Online]. Retrieved December 4, 2011, from www.pigeon.psy.tufts.edu/avc

Cook, R. G., \& Katz, J. S. (1999). Dynamic object perception by pigeons. Journal of Experimental Psychology: Animal Behavior Processes, 25, 194-210.

Friedman, A., Spetch, M. L., \& Lank, I. (2003). An automated apparatus for presenting depth-rotated three-dimensional objects in human and animal object recognition research. Behavior Research Methods, Instruments, \& Computers, 35, 343-349.

Jäger, R., \& Zeigler, H. P. (1991). Visual field organization and peck localization in the pigeon (Columba livia). Behavioural Brain Research, 45, 65-69.

Loidolt, M., Aust, U., Steurer, M., Troje, N. F., \& Huber, L. (2006). Limits of dynamic object perception in pigeons: dynamic stimulus presentation does not enhance perception and discrimination of complex shape. Learning \& Behavior, 34, 71-85.

Saxby, G. (2004). Practical holography (3rd ed.). Bristol, U.K.: Institute of Physics.

Ushitani, T., \& Fujita, K. (2005). Pigeons do not perceptually complete partly occluded photos of food: An ecological approach to the "pigeon problem. Behavioural Processes, 69, 67-78. 